\title{
Modification of Substrate Inhibition of Synaptosomal Acetylcholinesterase by Cardiotoxins
}

\author{
Seyed-Omid Ranaei-Siadat, Gholam-Hosein Riazi, Mehdi Sadeghi', Long-Sen Chang \\ Peyman Eghtesadi-Araghi, Gholam Hossein Hakimelahi" and Ali Akbar Moosavi-Movahedi* \\ Institute of Biochemistry and Biophysics, University of Tehran, Tehran, Iran \\ National Centre for Genetic Eng. \& Biotechnology, Tehran, Iran \\ ${ }^{*}$ Institute of Biomedical Sciences, National Sun Yat-Sen University, Kaohsiung, Taiwan \\ ${ }^{\S}$ School of Chemistry, Kaohsiung Medical University, Kaohsiung, Taiwan \\ "Taigen Biotechnology, 7F, 138 Hsin Ming Rd, Neihu Dist., Taipei, Taiwan
}

Received 7 July 2003, Accepted 8 September 2003

\begin{abstract}
Different types of cardiotoxin (I-V and $n$ ) were isolated and purified from the venom of the Taiwan cobra (Naja naja atra). The effects of these cardiotoxins were studied on membrane-bound acetylcholinesterase, which was isolated from a sheep's brain cortex. The results showed that cardiotoxins I-III, $\mathbf{V}$, and $\mathbf{n}$ activated the enzyme by modification of substrate inhibition, but cardiotoxin IV's reaction was different. The inhibition and activation of acetylcholinesterase were linked to the functions of the hydrophobicity index, presence of a cationic cluster, and the accessible arginine residue. Our results indicate that Cardiotoxins have neither a cationic cluster nor an arginine residue in their surface area of loop I; therefore, in contrast to fasciculin, cardiotoxins are attached by loop II to the peripheral site of the enzyme. As a result, fasciculin seems to stabilize nonfunctional conformation, but cardiotoxins seem to stabilize the functional conformation of the enzyme. Based on our experimental and theoretical findings, similar secondary and tertiary structures of cardiotoxins and fasciculin seem to have an opposite function once they interact with acetylcholinesterase.
\end{abstract}

Keywords: Acetylcholinesterase, Cardiotoxins, Peripheral site, Substrate inhibition, Synaptosome

\footnotetext{
*To whom correspondence should be addressed.

Tel: 98-21-6409517; Fax: 98-21-6404680

E-mail: moosavi@ut.ac.ir
}

\section{Introduction}

Acetylcholinesterase (AChE) (EC 3.1.1.7) terminates transmission at cholinergic synapses by rapid hydrolysis of acetylcholine. The symptomatic treatment of diseases, whose etiology involves this neurotransmitter depletion, can be achieved by controlled AchE inhibition. Anticholinesterase agents are thus of therapeutic importance. They are under active consideration for managing Alzheimer's disease (Harel et al., 1993).

The AChE active site consists of a gorge that is $2 \mathrm{~nm}$ deep, which is lined with aromatic residues (Rosenberry et al., 1996, De Ferrari et al., 2001). The gorge contains two sites of ligand binding - an acylation site near the base of the gorge and a peripheral site at the mouth of the gorge, about 10-20 from the acylation site. The acylation site consists of two subsites - a catalytic triad characteristic of serine hydrolases and an anionic subsite, where the positively-charged quaternary nitrogen of acetylcholine binds. The AChE peripheral site is an attractive target for the design of new classes of therapeutic agents. It is, therefore, important to understand how ligand binding to the peripheral site affects substrate hydrolysis (Mallender et al., 1999). Several ligands that bind exclusively to the peripheral site (for example propodium and fasciculin (FAS)) inhibit substrate hydrolysis at the acylation site, but the mechanistic interpretation of the inhibition is still unclear (Szegletes et al., 1998). Conformational interaction between the acylation and peripheral sites was recently found to modulate ligand affinities (Johnson et al., 2003). FAS is a member of the threefingered peptide toxin superfamily with 61 amino acid residues that were isolated from snake venom. This peptide inhibits mammalian and fish $\mathrm{AChE}$ by binding to the peripheral site of the enzyme. FAS is a selective peptide 
inhibitor of AChE, but not butyrylcholinesterase. The proposed mechanisms for AChE inhibition by FAS include allosteric events, resulting in the altered conformation of the AChE active center gorge (Sentjurc et al., 1999, Radic and Taylor, 2001). A comparison of the amino acid sequence of 175 snake toxins that are homologous to fasciculin showed that $\operatorname{Arg} 11$ and $\operatorname{Arg} 27$ are unique for fasciculin and no other toxin has arginine residues in the corresponding positions. Cardiotoxins (CTXs) are the best known of these toxins. Modification of the two unique arginine residues has a large effect and decreases activity by $73 \%$ (Arg11) and $85 \%$ (Arg27). They are located on the same side of the FAS molecule (Cervenansky et al., 1995).

Both acetylcholine and acetylthiocholine show substrate inhibition for $\mathrm{AChE}$ at high substrate concentrations. Substrate inhibition is due to the binding of acetylcholine or acetylthiocholine at the peripheral anionic site (or peripheral site) (Radic et al., 1991). This characteristic is useful for identifying AChE (EC 3.1.1.7) from butyrylcholinesterase (EC 3.1.1.8) and other esterases (Augustinsson, 1963). Cationic substrates can form a low-affinity complex at the peripheral site that accelerates catalytic hydrolysis at low substrate concentrations, but results in substrate inhibition at high concentrations because of the steric blockade of the product release (Szegletes et al., 1999). In AChE, the mutants of an Asp74 at the gorge entrance abolished substrate inhibition, possibly by inducing a conformational change. Mutation of a gorge entrance trytophan residue Trp-286 Ala is reported to reduce substrate inhibition (Shafferman et al., 1992). Trp286 is involved in the binding of quaternary ammonium at the peripheral site of AchE (Schalk et al., 1994).

One segment of the gorge that may play a major role in gorge enlargement is the large omega loop (defined by the Cys69-Cys96 disulfide bond) (Shi et al., 2003). Trp86 is in the center of this loop. In AChE, Trp86 can occupy two conformational states. One is functional as the anionic subsite of the acylation site, while the other hinders access to the acylation site and should reduce catalytic efficiency. Although the conformational flexibility of Trp86 and its effects on the catalytic activity provide a possible mechanism for the cross talk between amino acid residues, composing the peripheral site and the active center, the relay path of the allosteric signal is still unclear (Ordentlich et al., 1995). When Ala is substituted for Trp86 of AChE, FAS becomes an allosteric activator instead of an inhibitor. It is proposed that the allosteric modulation of AChE activity, induced by binding to amino acid residues within the peripheral anionic site, proceeds through this conformational change of Trp86 from a functional anionic subsite state to one that restricts the access of substrates to the active center (Radic et al., 1995).

On the other hand, the quaternary nitrogen groups of substrates and other active center ligands are stabilized by the cation-aromatic interaction with Trp86 rather than by ionic interactions (Ordentlich et al., 1995). The side chains of Trp86 subtend the quaternary ammonium-binding locus in the active site of AChE, while the bisquaternary ligand decamethonium is oriented along the narrow gorge leading to the active site. One quaternary group is appeased to the indole of Trp86 and the other to that of Trp286, near the top of the gorge. Structural and chemical data show the important role of aromatic groups as binding sites for quaternary ligands. They also provide complementary evidence assigning $\operatorname{Trp} 86$ to the "anionic" subsite of the active site and Trp286 to the "peripheral" anionic site (Harel et al., 1993).

Cardiotoxins (CTXs) or cytotoxins, neurotoxins, and phospholipase A2 are three major classes of toxic components that are present in the Taiwan cobra, Naja naja atra of the Elapidae family. CTXs are a group of major polypeptides of around 60 amino acid residues that comprise about $45-55 \%$ of the crude venom of the Taiwan cobra (Chiou et al., 1995a). Snake CTXs have a high basic (pI > 10) small molecular weight (approximately $6.5 \mathrm{kDa}$ ), all beta-sheet proteins. They exhibit a broad spectrum of interesting biological activities. The secondary structural elements in these toxins include antiparallel double- and triple-stranded beta-sheets. The threedimensional structures of these toxins reveal a unique asymmetric distribution of hydrophobic and hydrophilic amino acids (Kumar et al., 1997). CTXs and FAS possess distinct pharmacological and biochemical properties, despite the existence of a grossly similar tertiary structure among these toxins (i. e., a core consisting of a series of short loops and four disulfide bridges (Chiou et al., 1995b)).

There are two differing reports regarding the effect of just one type of CTX on AchE (Lin et al., 1977, Radic et al., 1995). In this paper, we studied the kinetic and structural views of six different types of CTXs (I-V, n) and their effects on AchE. We also examined a theoretical structural survey of FAS in order to investigate the mechanism of the AChE active site.

\section{Material and Methods}

Materials Sucrose, sodium phosphate monobasic, sodium phosphate bibasic, and sodium bicarbonate were obtained from Merck (Whitehouse Station, USA). Acetylthiocholine was from Fluka Co (Buchs, Switzerland). The 5,5' Dithiobis-(2-nitrobenzoic acid) or DTNB was received from Sigma (St. Louis, USA).

Cardiotoxins preparation procedure The chromatographic separation of cardiotoxins from Naja naja atra (Taiwan cobra) venom is similar to the procedure described by Chang (Chang et al., 1997). Lyophilized venom (5 g) was dissolved in $10 \mathrm{ml}$ of $1 \%$ acetic acid and then applied to a column of Sephadex G-50 $(2 \times 200$ $\mathrm{cm})$ at room temperature. The Sephadex column was eluted with $1 \%$ acetic acid at a flow rate of $40 \mathrm{ml} / \mathrm{min}$. Fractions of $15 \mathrm{ml}$ each were collected. The fractions containing proteins with molecular masses of lower than $20 \mathrm{kDa}$ were pooled and lyophilized for further purification. The venom proteins that were collected from the Sephadex G-50 column were applied to a Sp-Sephadex C-25 
column $(2 \times 95 \mathrm{~cm})$ that was equilibrated with a $0.005 \mathrm{M}$ sodium phosphate buffer $(\mathrm{pH} 7.0)$. The column was washed with the starting buffer $(0.005 \mathrm{M}$ phosphate buffer, $\mathrm{pH} 7.0)$ and then eluted with a salt gradient of $2,400 \mathrm{ml}$ from 0.0 to $0.5 \mathrm{M} \mathrm{NaCl}$ in the same buffer. Fraction 11 was subjected to re-chromatography on a Sephadex C-25 column $(2 \times 95 \mathrm{~cm})$ using a linear gradient of 2,000 $\mathrm{ml}$ of $0.5 \mathrm{M}$ to $1 \mathrm{M}$ ammonium acetate ( $\mathrm{pH}$ 5.8). CTXs $\mathrm{n}$ and I were separated from this fraction. Fractions 12 to 14 containing CTXs were further purified using reverse phase HPLC on a synchropak RP-P column $(4.6 \mathrm{~mm} \times 25 \mathrm{~cm})$. Fractions 12 and 13 contained cardiotoxins II and III, respectively. Fraction 14 was separated into cardiotoxin IV and cardiotoxin $\mathrm{V}$ using reverse phase HPLC that was eluted by linear gradient of $30-42 \%$ acetonitrile for $60 \mathrm{~min}$ at a flow rate of $1 \mathrm{ml} / \mathrm{min}$.

Animals Adult sheep were killed by rapid cervical dislocation in Tehran's slaughterhouse (Ghoche-hesar, Ramandi 4), Iran.

\begin{abstract}
Synaptosome isolation procedure Nerve endings were prepared by Dodd's method (Dodd et al., 1981). A Beckman L5-50 ultracentrifuge was used. Preparation was at $4^{\circ} \mathrm{C}$ throughout. Sheep of either sex were killed by cervical dislocation and the whole brain was placed in an ice-cold $0.32 \mathrm{M}$ sucrose solution. The cerebral cortices (weighing approximately $50 \mathrm{~g}$ each) were rapidly removed and placed in a 10 volume ice-cold unbuffered $0.32 \mathrm{M}$ sucrose solution. A motor-driven potter teflon glass homogenizer was used to disperse the tissues in the solution at a motor speed of $800 \mathrm{rpm}$ and 12 strokes of the pestle. The initial homogenate was centrifuged at $5100 \mathrm{rpm}$ in MSE centrifuge for approximately $30 \mathrm{~min}$. The supernatant was placed on top of $10 \mathrm{ml}$ of $1.2 \mathrm{M}$ sucrose and then centrifuged at $35000 \mathrm{rpm}$ for $71 \mathrm{~min}$ by a Beckman L5-50 ultracentrifuge. The pellet was carefully collected and suspended in $160 \mathrm{ml}$ of $0.32 \mathrm{M}$ sucrose. The temperature was kept ice cold during the preparation. Synaptosomes were snap-frozen in liquid nitrogen and then rapidly brought to room temperature.
\end{abstract}

Assay of acetylcholinesterase activity The acetylcholinesterase activity was assayed using a lambda $2 \mathrm{UV} /$ visible spectrophotometer (Perkin-Elmer, Norwalk, USA), according to Ellmans procedure (Ellman et al., 1961). Acetylthiocholine was used as the substrate. The enzyme activity was monitored at $412 \mathrm{~nm}$ by following the increase of yellow color that was produced from thiocholine when it reacted with DTNB. The reaction mixture contained Acetylthiocholine, DTNB, and a synaptosom solution in a $0.1 \mathrm{M}$ potassium phosphate buffer ( $\mathrm{pH}$ 7.4). The acetylthiocholine hydrolysis reaction was initiated by the addition of the synaptosomal solution $(50 \mu \mathrm{l})$ to the sample cuvette. Ten seconds after mixing, the increase in the absorbance of the mixing solution at $412 \mathrm{~nm}$ was monitored. A linear plot of the absorbance versus time was recorded for $2 \mathrm{~min}$. The acetylcholinesterase activity was calculated on the basis of the extinction coefficient of the yellow anion $(13.6 / \mathrm{mM} \mathrm{cm})$. Data were analyzed using SigmaPlot 8.0 (SPSS, Inc. Chicago, USA). Activities of the AChE - CTXs complexes were measured during a 10-min incubation of $640 \mathrm{nM}$ AChE with $1.3 \mu \mathrm{M}$ CTXs to form the complex.

Hydrophobicity profile The hydrophobicity profile is a simple way to qualify the concentration of the hydrophobic residue along the linear polypeptide chain. The hydrophobicity profile of CTXs and FAS were carried out on ProtScale software (http:// www.expasy.ch/cgi-bin/protscale.pl), based on the Kyte and Doolittle hydropathy scale (Kyte and Doolittle, 1982). A N = 9 size window was run along the length of the toxin sequences. Plotting the curve revealed the loci of minima and maxima in hydrophobicity along the linear polypeptide chain.

Accessible surface area (ASA) The accessible surface area (ASA) for individual atoms of the proteins was calculated from the atomic coordinates that were deposited in the protein databank (PDB) (Berman et al., 2000). For each atom, a sufficiently large number of approximately evenly-distributed points were placed on the salvation sphere of radius $\mathrm{Ra}+\mathrm{Rw}$ that was centered at the atomic position, where Ra and Rw are the Van der Waals radii of atom A and solvent probe, respectively (Naderi-Manesh et al., 2001).

\section{Results}

Substrate inhibition The steady-state kinetics of $\mathrm{AChE}$ in the presence of acetylthiocholine (as substrate) exhibits the substrate inhibition, which is shown in Fig. 1 as the Lineweaver-Burk plots of the effect of CTXs on AChE and substrate inhibition phenomena. $K_{m}$ of AChE was $82 \mu \mathrm{M}$ and $V_{\max }$ was $53 \mu \mathrm{M} / \mathrm{min} / \mathrm{mg}$ protein. In the presence of CTXs (I$\mathrm{V}$ and n) $K_{m}$ s were $124,140,144,181,106$, and $139 \mu \mathrm{M}$ and $V_{\max }$ s were $59,60,60,77,52$, and $61 \mu \mathrm{M} / \mathrm{min} / \mathrm{mg}$ protein, respectively (Table 1). AChE in the presence of CTX IV (and in the absence of the remaining CTXs) showed substrate inhibition; whereas, in the presence of the remaining CTXs (and in the absence of CTX IV), the substrate inhibition disappeared. $K_{i}$ for $\mathrm{AChE}$ and the AChE/CTX IV complex were 0.84 and $1 \mathrm{mM}$, respectively. As shown in Fig. 1, the Lineweaver-Burk plot of the data displays a non-linear curve over the substrate concentration range beyond the initial inhibition.

\section{Hydrophobicity profile and index $(\mathrm{H} \varphi)$ of CTXs and FAS} Figure 2 shows the hydrophobicity profile of CTXs and FAS. Loop II is the most important portion of these profiles. The absence of the substrate inhibition of AChE is dependent on the hydrophobicity index $(\mathrm{H} \varphi)$ of CTXs. H $\varphi$ s are 0.157, $0.137,0.12,0.112,0.105,-0.03$, and -0.79 for CTXs n, III, I, II, V, IV, and FAS, respectively (Table 2) (amino acid sequences from Chang et al., 2002). Among cardiotoxins, only type IV with a lower hydrophobicity index $(-0.03)$ showed some substrate inhibition (Kyte and Doolittle, 1982).

ASA for CTXs and FAS Tables 3 and 4 show the comparative ASA values for each positive residue in CTXs and FAS. The structure references of the calculations were as follows: CTX I (2CDX) (Janke et al. 1994), CTX II (1CRE) (Bhaskaran et al., 1994a), CTX III (2CRT) (Bhaskaran et al., 1994b), CTX IV (1KBS) (Jang et al., 1997), CTX V (1CHV) (Jayaraman et al., 1994), and FAS (1FSC) (LeDu et al., 1996). 

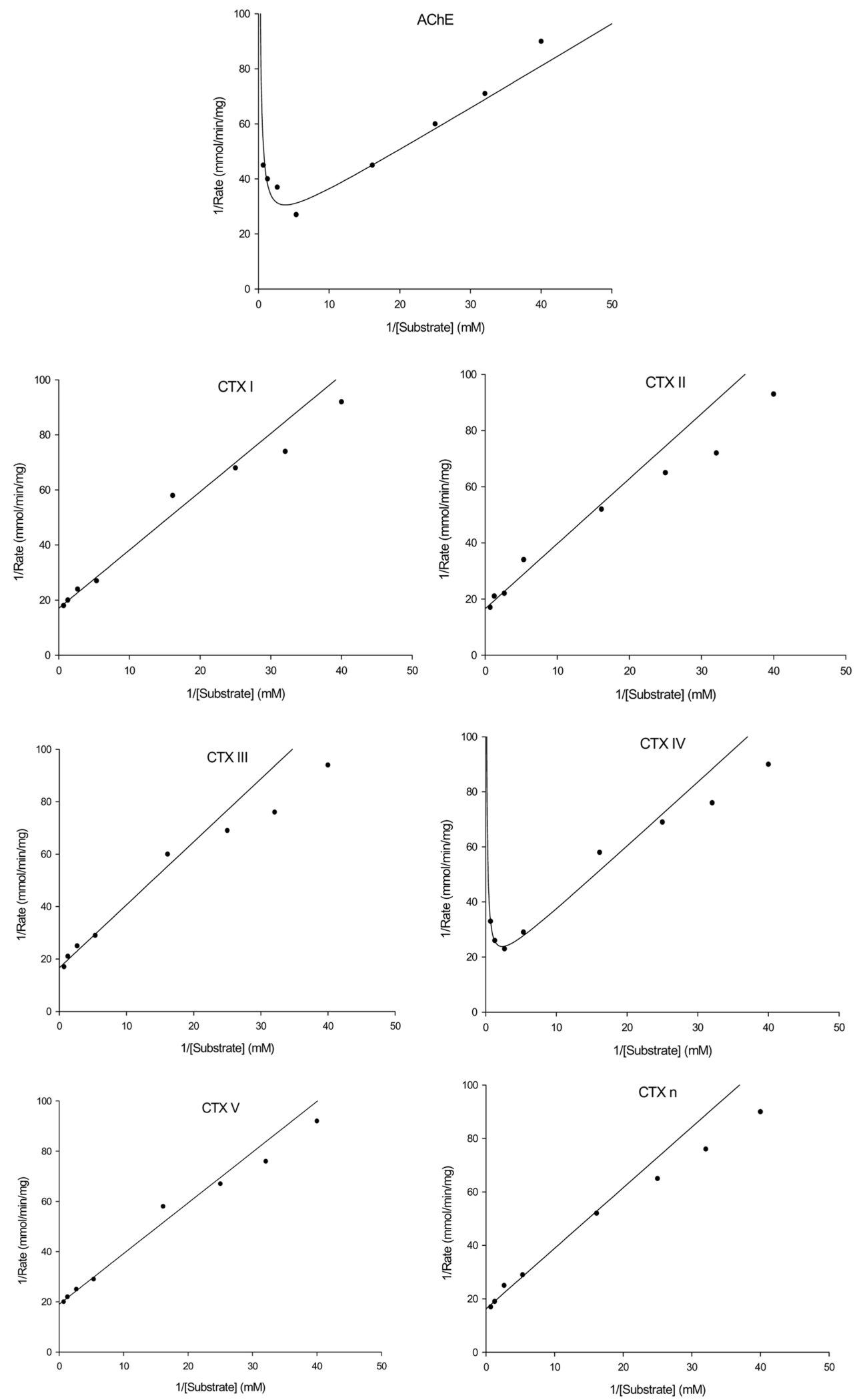

Fig. 1. Lineweaver-Burk plots of AChE in the absence and presence of CTXs (I, II, III, IV, V, and n). Substrate concentration ranges were from $0.025-1.5 \mathrm{mM}$ for the substrate inhibition study and $0.1 \mathrm{mM}$ of acetylthiocholin for the steady state. 
Table 1. Kinetic parameters for AChE with and without CTXs. $V_{\max }(\mu \mathrm{M} / \mathrm{min} / \mathrm{mg})$ and $K_{m}, K_{i}(\mu \mathrm{M})$

\begin{tabular}{ccccrrrr}
\hline & AChE & \multicolumn{1}{c}{ CTX I } & CTX II & CTX III & CTX IV & CTX V & CTX n \\
\hline$V_{\max }$ & $53 \pm 13$ & $59 \pm 1.9$ & $60 \pm 3.7$ & $60 \pm 3$ & $77 \pm 10$ & $52 \pm 1.3$ & $61 \pm 2.9$ \\
$K_{m}$ & $82 \pm 36$ & $124 \pm 10$ & $140 \pm 29$ & $144 \pm 26$ & $181 \pm 40$ & $106 \pm 9.1$ & $139 \pm 2.1$ \\
$K_{i}$ & $840 \pm 46$ & & & & $1000 \pm 29$ & & \\
\hline
\end{tabular}
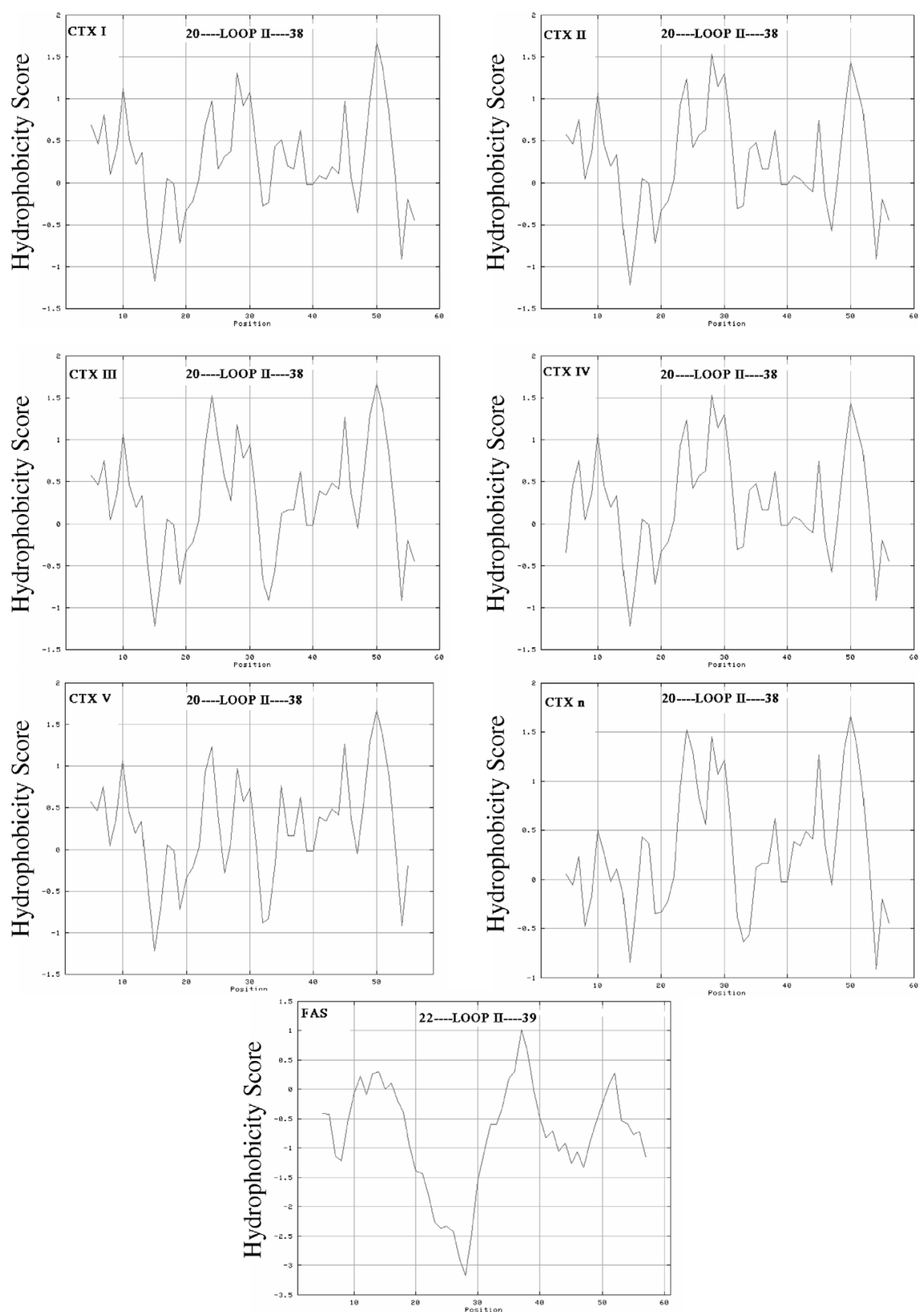

Fig. 2. Hydrophobicity profiles of CTXs I, II, III, IV, V, n, and fasciculin (FAS). Hydrophobicity score is the hydrophobicity value of each amino acid.

Interaction of Trp86 of AChE with Arg11 of FAS The distance calculation between the atomic coordination of FAS amino acid residues and Trp86 of AChE in the complex AChE-FAS (Bourne et al., 1995) indicates only the interaction between Arg11 of FAS and Trp86 of AChE.

\section{Discussion}

CTXs were selected and their effects studied on AChE because of their structural similarities with FAS (a selective inhibitor of peripheral site of AChE). The effect of CTXs, I-V, 
Table 2. Net charges, positive charges, arginine residues, lysine residues, positive charges in loop II, arginine residues in loop II, lysine residues in loop II, Aliphatic index, Hydrophobicity indexes $(\mathrm{H} \varphi)$, pI of CTXs I, II, III, IV, V, n and FAS

\begin{tabular}{cccccccccccc}
\hline & $\begin{array}{c}\text { Net } \\
\text { charges }\end{array}$ & $\begin{array}{c}\text { positive } \\
\text { charges }\end{array}$ & $\begin{array}{c}\text { arginine } \\
\text { residues }\end{array}$ & $\begin{array}{c}\text { lysine } \\
\text { residues }\end{array}$ & $\begin{array}{c}\text { positive } \\
\text { charges } \\
\text { in loop II }\end{array}$ & $\begin{array}{c}\text { arginine } \\
\text { residues in } \\
\text { loop II }\end{array}$ & $\begin{array}{c}\text { lysine } \\
\text { residues in } \\
\text { loop II }\end{array}$ & $\begin{array}{c}\text { Aliphatic } \\
\text { index }\end{array}$ & H $\varphi$ & pI \\
\hline FAS & $4+$ & 9 & 5 & 4 & 6 & 4 & 2 & 36.72 & -0.79 & 8.7 \\
CTX I & $7+$ & 10 & 2 & 8 & 3 & 1 & 2 & 87.67 & 0.12 & 9.2 \\
CTX II & $8+$ & 10 & 2 & 8 & 3 & 1 & 2 & 82.67 & 0.112 & 9.3 \\
CTX III & $9+$ & 11 & 2 & 9 & 4 & 1 & 3 & 82.67 & 0.137 & 9.4 \\
CTX IV & $9+$ & 11 & 3 & 8 & 4 & 1 & 3 & 76.17 & -0.027 & 9.4 \\
CTX V & $9+$ & 11 & 2 & 9 & 4 & 1 & 3 & 81 & 0.105 & 9.4 \\
CTX n & $8+$ & 10 & 2 & 8 & 4 & 1 & 3 & 81.17 & 0.157 & 9.3 \\
\hline
\end{tabular}

Table 3. Accessible surface area (ASA) values of positive residues (arginine and lysine) of CTXs I, II, III, IV, and V

\begin{tabular}{|c|c|c|c|c|c|}
\hline Amino acid residue & CTX I ASA & CTX II ASA & CTX III ASA & CTX IV ASA & CTX V ASA \\
\hline $\operatorname{Arg} 1$ & & & & 165 & \\
\hline Lys2 & 89 & 128 & 109 & 101 & 110 \\
\hline Lys5 & 132 & 97 & 81 & 109 & 89 \\
\hline Lys 12 & 143 & 162 & 101 & 133 & 114 \\
\hline Lys 18 & 45 & 105 & 87 & 110 & 72 \\
\hline Lys 23 & 54 & 38 & 51 & 52 & 4 \\
\hline Lys30 & & & & & 170 \\
\hline Lys31 & & & 174 & & \\
\hline Lys35 & 129 & 102 & 125 & 130 & 117 \\
\hline $\operatorname{Arg} 36$ & 44 & 46 & 72 & 20 & 48 \\
\hline Lys44 & 198 & 173 & 133 & 179 & 184 \\
\hline Lys50 & 102 & 79 & 115 & 97 & 99 \\
\hline $\operatorname{Arg} 58$ & 143 & 226 & 219 & 131 & 215 \\
\hline
\end{tabular}

Table 4. Accessible surface area (ASA) values of positive residues (arginine and lysine) of FAS

\begin{tabular}{ccc}
\hline Amino acid residue & ASA in free FAS & $\begin{array}{c}\text { ASA in FAS in } \\
\text { complex with AChE }\end{array}$ \\
\hline Arg11 & 213 & 96 \\
Arg24 & 52 & 36 \\
Lys25 & 54 & 49 \\
Arg27 & 67 & 22 \\
Arg28 & 120 & 84 \\
Lys32 & 148 & 80 \\
Arg37 & 32 & 4 \\
Lys51 & 73 & 81 \\
Lys58 & 96 & 101 \\
\hline
\end{tabular}

and n, (except CTX IV) showed modification of the substrate inhibition on AChE. The variation of the substrate inhibition seemed to be dependent on H $\varphi$ of CTXs, because only CTX IV with a lower $\mathrm{H} \varphi$ showed a small substrate inhibition. The Lineweaver-Burk plots of the data displayed non-linear curves and a non-Michaelis kinetic for the AChE in the absence of CTXs and the presence of CTX IV (Fig. 1). Studies on the substrate concentrations and enzyme activities by the
Lineweaver-Burk method showed two stages: (1) The enzyme activity of low substrate concentrations decreased in the presence of CTXs. (2) The enzyme activity in the presence of CTXs in high substrate concentrations showed no substrate inhibition. The interpretations regarding these two stages are as follows: (1) Loop II was postulated to be intricately involved in the biological activity of CTXs (Kumar et al., 1997). This loop had a larger score of hydrophobicity and nearly 3-4 positive charges (including one Arg) (see Figs. 2 and 4) for the approximation of CTXs with the peripheral site (also called peripheral anionic site (PAS)). On the other hand, the peripheral site of $\mathrm{AChE}$ is also named the "Aromatic gorge" because nearly $40 \%$ of its lining is composed of a ring consisting of 14 conserved aromatic amino acids (Harel et al., 1993) and many other hydrophobic residues. It is our opinion that loop II on the CTXs can interact with the peripheral site of AChE through a hydrophobic interaction. This interaction could be the cause of prohibiting the easy (normal) entrance of the substrate to the active site gorge. Thus, the enzyme activity is being decreased in lower substrate concentrations, and (2), according to the steric blockage model (Rosenberry et al., 1999), in higher concentrations, the substrates bind to the peripheral site of $\mathrm{AChE}$ and block the product release. This accounts for the phenomenon of substrate inhibition. 

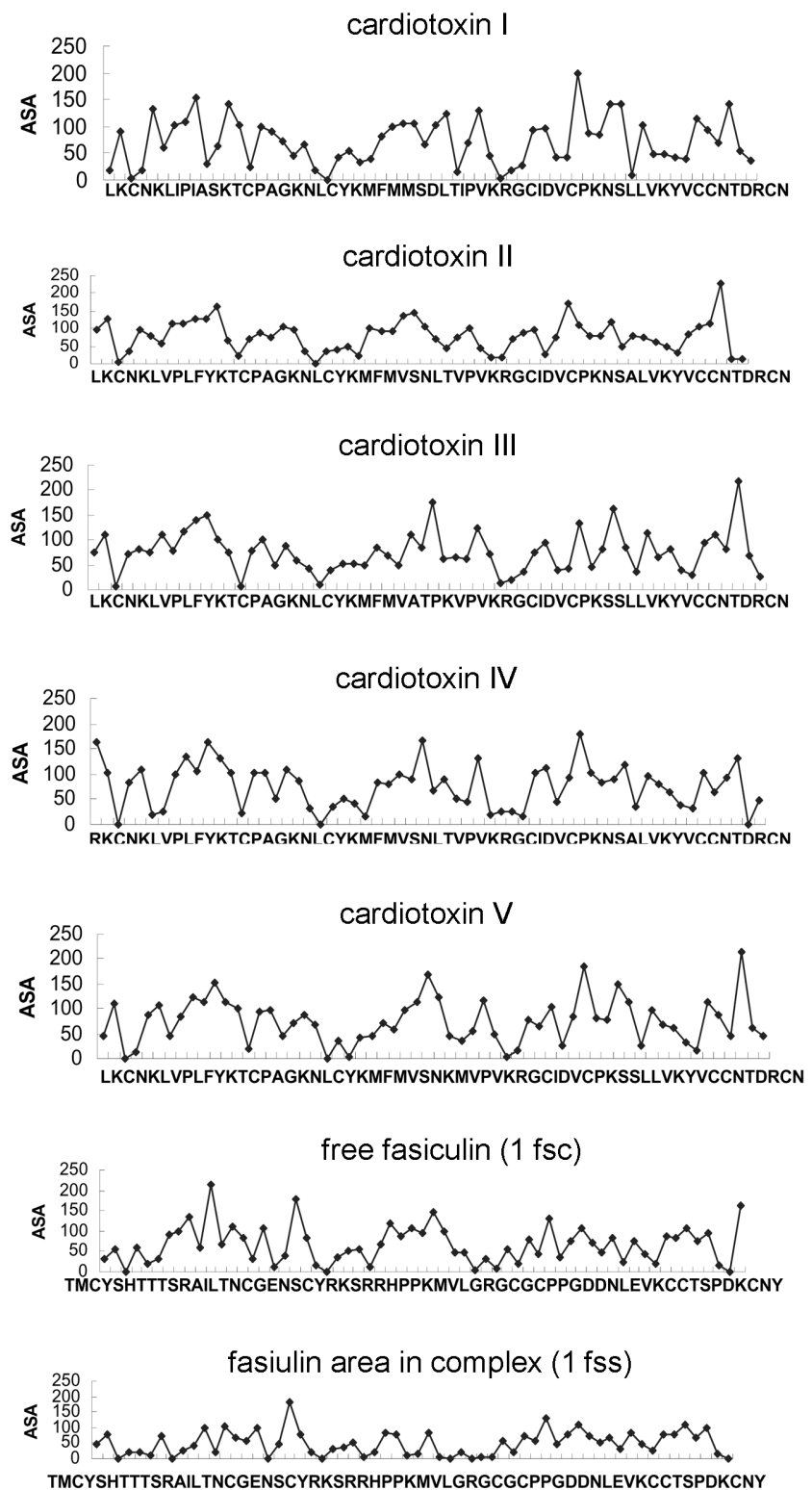

Fig. 3. Accessible surface area (ASA) of CTXs I, II, III, IV, V, and FAS. (Crystallographic data of CTX $n$ is not in PDB).

Furthermore, it is believed that in higher substrate concentrations, CTXs occupy the peripheral site of AChE (location of binding of substrate in higher concentrations for the formation of steric blockage model and occurring of substrate inhibition) causing the disappearance of the substrate inhibition.

FAS is a selective inhibitor of the peripheral site of AChE. FAS was selected because of its suitable inhibitory model to study the mechanism of the CTXs-AChE complexes. FAS has four arginine residues; a positive cluster, a hydrophobic cluster (including Pro30 and Pro31) at loop II, and one accessible arginine residue $\operatorname{Arg} 11$ (ASA is equal to 213, Table 4) on the tip of loop I (Figs. $3 \& 4$ and Table 2). A comparison of the amino acid sequence of 175 snake toxins that are homologous with FAS showed that $\operatorname{Arg} 11$ and $\operatorname{Arg} 27$ are unique for FAS. FAS via Pro30, Pro 31, and Arg27 of loop II can interact with Ile30, Leu289, and Trp286 of AChE, respectively (Bourne et al., 1995). We calculated the distance between all of the amino acid residues of FAS with Trp86 of AChE from the crystallographic data (Bourne et al., 1995). The results showed that the best interaction ( $7 \AA$ ) belonged to $\operatorname{Arg} 11$ on loop I of FAS with Trp86 of AChE. Thus, Arg11 of FAS and Trp86 of AChE are the two key amino acid residues for the inhibition of AChE by FAS. Previous studies also showed that the modification of the Arg11 residue decreased AChE activity by $73 \%$ (Cervenansky et al., 1995); however, when Ala was substituted for Trp86 of AChE, FAS became allosterictly an activator instead of an inhibitor (Radic et al., 1995). For the inhibition of AChE, the ligand must interact with $\operatorname{Trp} 286$ at the peripheral site and with $\operatorname{Trp} 86$ at the rim (Harel et al., 1993). Cation- $\pi$ interaction is an essential element for these interactions (Harel et al., 1993). The tip of loop II of CTXs consists of hydrophobic amino acids and it can interact with the peripheral site of AchE, where the tip of loop I contains no arginine. It was suggested that amino acid Trp86 could not be connected to loop I on CTXs. FAS (in Trp86 Ala mutation) (Radic et al., 1995) and CTXs cause a very small inhibition of $\mathrm{AChE}$ in low substrate concentrations and the activation of the enzyme in high concentrations of the substrate. The reason for this phenomenon may be the interaction of just one loop (loop II) of toxin to AChE. Thus, FAS causes the blocking of Trp86, and CTXs hold Trp86 of AChE in the free stage. The functional and non-functional conformations of AChE are related to Trp86 (Radic et al., 1995). We believe that the arginine side chain, in comparison with the lysine side chain, plays an essential role in the inhibition of $\mathrm{AChE}$ because of the similarities of the space volume of the arginine side chain (guanidino group) with quaternary ammonium cationic substrates and the inhibitors of AChE. On the other hand, when fasciculin is present, the gorge width distribution is altered and the gorge is more likely to be narrow (Tai et al., 2002). Our theoretical data showed that in the presence of CTXs, the gorge width was altered and the gorge was more likely to be broader (data not shown).

AChE in the presence of the CTX type IV shows substrate inhibition. CTX IV has a cationic cluster that is comprised of positively-charged residues on the concave side of the molecule. CTX IV and CTX II differ in their amino acid sequence by a single amino acid at the $\mathrm{N}$-terminal end. Leucine at the N-terminal end of CTX II is replaced by the arginine residue in CTX IV (Fig. 4). CTX IV is a unique snake venom with a positively-charged residue at the $\mathrm{N}$ terminal amino acid. All of the other CTXs have a hydrophobic amino acid (leucine or isoleucine) at their $\mathrm{N}$ terminal end. Similar clusters, consisting of positively-charged residues are not found in other CTXs (Jang et al., 1995). Therefore, only CTX IV, in addition to a hydrophobic loop (loop II), has a positive cluster. We believe this positive cluster is an anchoring site for the substrate for the formation of a 


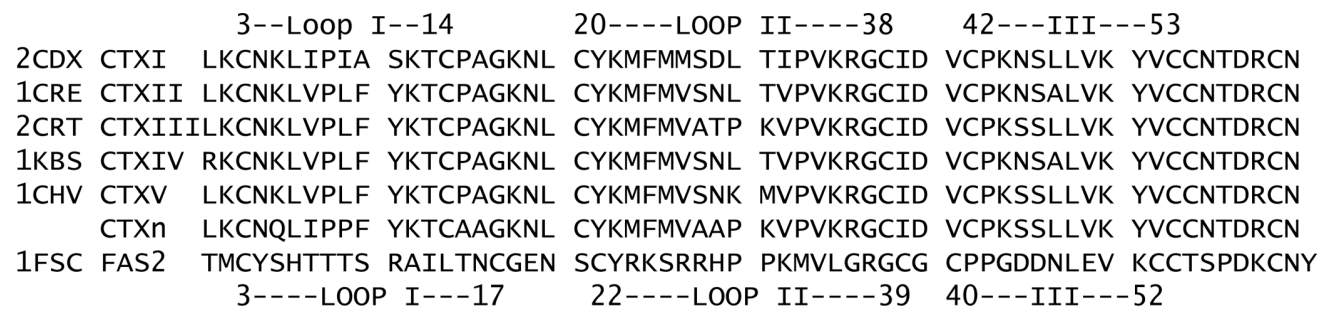

Fig 4. Comparison of the sequence of CTXs I, II, III, IV, V, n, and FAS. The amino acid residues \#3-14, \#20-38, and \#42-53 of CTXs correspond to loops I, II, and III, respectively. The amino acid residues \#3-17, \#22-39, and \#40-52 of FAS correspond to loops I, II, and III, respectively. 2CDX, 1CRE, 2CRT, 1KBS, 1CHV, 1FSC are from the protein databank (PDB) ID for CTXs I- V, and FAS, respectively. CTX $n$ has no PDB ID. FAS has one more amino acid than CTXs.

steric blockage model. In addition, CTX IV has the lowest amount of $\mathrm{H} \varphi$ of CTXs and cannot be compared with high substrate concentrations in the interaction with the peripheral site. This is the reason for the small amount of substrate inhibition upon the interaction of AChE with CTX IV.

FAS has hydrophobic and hydrophilic clusters at loop II (Fig. 2); whereas, CTXs have just a hydrophobic cluster without a cationic cluster at loop II (Fig. 2). On the other hand, FAS has 5 arginine residues. CTXs I, II, III, V, and n have 2 arginine residues, and CTX IV has 3 arginine residues (Table 2). It is extremely important to note that FAS with the lowest $\mathrm{H} \varphi$ (possession of a cationic cluster, and possession of five (5) Arg) shows an inhibitory effect on AchE. However; CTXs I, II, III, V, and n (without a cationic cluster and with only two (2) Arg and a higher $\mathrm{H} \varphi$ ) are the activators of AchE. Also, CTX IV that has the lowest $\mathrm{H} \varphi$ of CTXs with a cationic cluster shows a minimal disappearance of substrate inhibition. Accordingly, a "cationic" cluster that is comprised of positively-charged residues on the concave side of the molecule, the number and the location of Arg residues, and a low $\mathrm{H} \varphi$ is necessary for the enzyme inhibition. The AchE inhibition is dependent on the following factors: (1) the hydrophobicity of the ligand, as well as (2) the number, position of tertiary structure, and types of basic amino acids. A comparison of the curves of the hydrophobicity profiles and ASA show the parts of the hydrophobic centers that interact with AChE. Tables 3 and 4 show ASA in the CTXs and FAS contribution as the result of its positive-charge groups. The side chains of the Arg and Lys residues contribute to the formation of the cationic cluster on the protein surface. Our results showed the same behaviour in the free enzyme relative to the membrane-bound enzyme (data not shown).

In conclusion, FAS stabilizes the inactive conformation and CTXs stabilize the active conformation of AChE. Therefore, the existence of the positive charge and the hydrophobic clusters in loop II of the peptide are necessary for their interactions with the enzyme. It is clear that in addition to these clusters, an accessible arginine at loop I of the peptide that interacts with Trp86 of the enzyme helps the inhibition of AChE.

Acknowledgments The authors thank Dr. M. Fooladi for his valuable comments on the manuscript. The financial support of the Research Council of the University of Tehran is gratefully acknowledged.

\section{References}

Augustinsson, K. B. (1963) Cholinesterase and anticholinesterase agents; in Classification and Comparative Enzymology of the Cholinesterases and Methods for Their Determination. Eichler, O. and Farah, A. (eds.), pp. 89-128, Springer-Verlag, Berlin, Germany.

Berman, H. M., Westbrook, J., Feng, Z., Gilliland, G., Bhat, T. N., Weissig, H., Shindyalov, I. N. and Bourne, P. E. (2000) The Protein Data Bank. Nucleic Acids Res. 28, 235-242.

Bhaskaran, R., Huang, C. C., Tsai, Y. C., Jayaraman, G., Chang, D. K. and Yu, C. (1994a) Cardiotoxin II from Taiwan cobra venom, Naja naja atra. Structure in solution and comparison among homologous cardiotoxins. J. Biol. Chem. 269, 2350023508 .

Bhaskaran, R., Huang, C. C., Chang, K. D. and Yu, C. (1994b) Cardiotoxin III from Taiwan Cobra (Naja naja atra) Determination of Structure in Solution and Comparison with Short Neurotoxins. J. Mol. Biol. 235, 1291-1301.

Bourne, Y., Taylor, P. and Marchot, P. (1995) Acetylcholinesterase inhibition by fasciculin: crystal structure of the complex. Cell 83, 503-512.

Cervenansky, C., Engstrom, A. and Karlsson, E. (1995) Role of arginine residues for the activity of fasciculin. Eur. J. Biochem. 229, 270-275.

Chang, L. S., Chou, Y. C., Lin, S. R., Wu, B. N., Lin, J., Hong, E., Sun, Y. J. and Hsiao, C. D. (1997) A novel neurotoxin, cobrotoxin b, from Naja naja atra (Taiwan cobra) venom: purification, characterization, and gene organization. $J$. Biochem. (Tokyo). 122, 1252-1259.

Chang, L. S., Huang, H. B. and Lin, S. R. (2002) The multiplicity of cardiotoxins from Naja naja atra (Taiwan cobra) venom. Toxicon. 38, 1065-1076.

Chiou, S. H., Hung, C. C., Huang, H. C., Chen, S. T., Wang, K. T. and Yang, C. C. (1995a) Sequence comparison and computer modeling of cardiotoxins and cobrotoxin isolated from Taiwan cobra. Biochem. Biophys. Res. Commun. 206, 2232.

Chiou, S. H., Chuang, M. H., Hung, C. C., Huang, H. C., Chen, S. T., Wang, K. T. and Ho, C. L. (1995b) Inhibition of protein kinase $\mathrm{C}$ by snake venom toxins: comparison of enzyme 
inhibition, lethality and hemolysis among different cardiotoxin isoforms. Biochem. Mol. Biol. Int. 35, 1103-1112.

De Ferrari, G. V., Mallender, W. D., Inestrosa, N. C. and Rosenberry, T. L. (2001) Thioflavin T is a fluorescent probe of the acetylcholinesterase peripheral site that reveals conformational interactions between the peripheral and acylation sites. J. Biol. Chem. 276, 23282-23287.

Dodd, P. R., Hardy, J. A., Oakley, A. E., Edwardson, J. A., Perry, E. K. and Delaunoy, J. P. (1981) A rapid method for preparing synaptosomes: comparison, with alternative procedures. Brain Res. 226, 107-118.

Ellman, G. L., Courtney, K. D., Andres, V. Jr and Featherstone, R. M. (1961) A new rapid colorimetric determination of acetylcholinesterase activity. Biochem. Pharmacol. 7, 788-795.

Harel, M., Schalk, I., Ehret-Sabatier, L., Bouet, F., Goeldner, M., Hirth, C., Axelsen, P. H., Silman, I. and Sussman, J. L. (1993) Quaternary ligand binding to aromatic residues in the activesite gorge of acetylcholinesterase. Proc. Natl. Acad. Sci. USA. 90, 9031-9035.

Jahnke, W., Mierke, D. F., Beress, L. and Kessler, H. (1994) Structure of cobra cardiotoxin CTX I as derived from nuclear magnetic resonance spectroscopy and distance geometry calculations. J. Mol. Biol. 240, 445-458.

Jang, J. Y., Krishnaswamy, T., Kumar, S., Jayaraman, G., Yang, P. W. and Yu, C. (1997) Comparison of the hemolytic activity and solution structures of two snake venom cardiotoxin analogues which only differ in their $\mathrm{N}$-terminal amino acid. Biochemistry 36, 14635-14641.

Jayaraman, G., Kumar, T. K. S., Tsai, C. C., Chou, S. H., Ho, C. L. and Yu, C. (2000) Elucidation of the Solution Structure of Cardiotoxin Analogue V from the Taiwan Cobra (Naja naja atra) Venom. Protein Sci. 9, 637-646.

Johnson, J. L., Cusack, B., Davies, M. P., Fauq, A. and Rosenberry, T. L. (2003) Unmasking tandem site interaction in human acetylcholinesterase. Substrate activation with a cationic acetanilide substrate. Biochemistry 42, 5438-5452.

Kumar, T. K., Jayaraman, G., Lee, C. S., Arunkumar, A. I., Sivaraman, T., Samuel, D. and Yu, C. (1997) Snake venom cardiotoxins-structure, dynamics, function and folding. $J$. Biomol. Struct. Dyn. 15, 431-463.

Kyte, J. and Doolittle, R. F. (1982) A simple method for displaying the hydropathic character of a protein. J. Mol. Biol. 157, 105-132.

LeDu, M. H., Housset, D., Marchot, P., Bougis, P. E., Navaza, J. and FontecillaCamps, C. (1996) Structure of fasciculin 2 from green mamba snake venom: Evidence for unusual loop flexibility. Acta. Crystallogr. D. Biol. Crystallogr. 52, 87.

Lin, S. Y., Liao, C. and Lee, C. Y. (1977) Mechanism of anticholinesterase activities of cardiotoxin, protamine and polylysine. Biochem. J. 161, 229-232.

Mallender, W. D., Szegletes, T. and Rosenberry, T. L. (1999) Organophosphorylation of acetylcholinesterase in the Presence of Peripheral Site Ligands: distinct effects of propodium and fasciculin. J. Biol. Chem. 274, 8491-8499.

Naderi-Manesh, H., Sadeghi, M., Arab, S. and Moosavi Movahedi,
A. A. (2001) Prediction of protein surface accessibility with information theory. Proteins 42, 452-459.

Ordentlich, A., Barak, D., Kronman, C., Ariel, N., Segall, Y., Velan, B. and Shafferman, A. (1995) Contribution of aromatic moieties of tyrosine 133 and of the anionic subsite tryptophan 86 to catalytic efficiency and allosteric modulation of acetylcholinesterase. J. Biol. Chem. 270, 2080-2091.

Radic, Z., Reiner, E. and Taylor, P. (1991) Role of the peripheral anionic site on acetylcholinesterase: inhibition by substrates and coumarin derivatives. Mol. Pharmacol. 39, 98-104.

Radic, Z., Quinn, D. M., Vellom, D. C., Camp, S. and Taylor, P. (1995) Allosteric control of acetylcholinesterase catalysis by fasciculin. J. Biol. Chem. 270, 20391-20399.

Radic, Z. and Taylor, P. (2001) Interaction kinetics of reversible inhibitors and substrates with acetylcholinesterase and its fasciculin 2 complex. J. Biol. Chem. 276, 4622-4633.

Rosenberry, T. L., Rabl, C. R. and Neumann, E. (1996) Binding of the neurotoxin fasciculin 2 to the acetylcholinesterase peripheral site drastically reduces the association and dissociation rate constants for $\mathrm{N}$-methylacridinium binding to the active site. Biochemistry 35, 685-690.

Rosenberry, T. L., Mallender, W. D., Thomas, P. J. and Szegletes, T. (1999) A steric blockade model for inhibition of acetylcholinesterase by peripheral site ligands and substrate. Chem. Biol. Interact. 119, 85-89.

Schalk, I., Ehret-Sabatier, L., Bouet, F., Goeldner, M. and Hirth, C. (1994) Trp279 is involved in the binding of quaternary ammonium at the peripheral site of Torpedo marmorata acetylcholinesterase. Eur. J. Biochem. 219, 155-159.

Sentjurc, M., Pecar, S., Stojan, J., Marchot, P., Radic, Z. and Grubic, Z. (1999) Electron paramagnetic resonance reveals altered topography of the active center gorge of acetylcholinesterase after binding of fasciculin to the peripheral site. Biochim. Biophys. Acta 1430, 349-358.

Shafferman, A., Velan, B., Ordentlich, A., Kronman, C., Grosfeld, H., Leitner, M., Flashner, Y., Cohen, S., Barak, D. and Ariel, N. (1992) Substrate inhibition of acetylcholinesterase: residues affecting signal transduction from the surface to the catalytic center. EMBO J. 11, 3561-3568.

Shi, J., Tai, K., McCammon, A. J., Taylor, P. and Johnson, D.A. (2003) Nanosecond dynamics of the mouse acetylcholinesterase Cys69-Cys96 Omega loop. J. Biol. Chem. 278, 30905-30911.

Szegletes, T., Mallender, W. D. and Rosenberry, T. L. (1998) Nonequilibrium analysis alters the mechanistic interpretation of inhibition of acetylcholinesterase by peripheral site ligands. Biochemistry 37, 4206-4216.

Szegletes, T., Mallender, W. D., Thomas, P. J. and Rosenberry, T. L. (1999) Substrate binding to the peripheral site of acetylcholinesterase initiates enzymatic catalysis. Substrate inhibition arises as a secondary effect. Biochemistry 38, 122133.

Tai, K., Shen, T., Henchman, R. H., Bourne, Y., Marchot, P. and McCammon, J. A. (2002) Mechanism of acetylcholinesterase inhibition by fasciculin: a 5-ns molecular dynamics simulation. J. Am. Chem. Soc. 29, 6153-6161. 